\section{CONGENITAL HEART DISEASE}

\section{Heart disease and pregnancy}

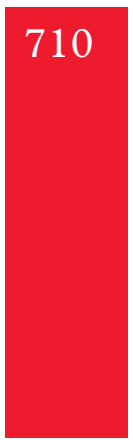

Samuel C Siu, Jack M Colman

Toronto, Ontario, Canada

$\mathrm{P}$
University of Toronto Congenital Cardiac Centre for Adults,

University Health Network and Mount Sinai Hospital,

regnancy in most women with heart disease has a favourable maternal and fetal outcome. With the exception of patients with Eisenmenger syndrome, pulmonary vascular obstructive disease, and Marfan syndrome with aortopathy, maternal death during pregnancy in women with heart disease is rare. ${ }^{1-4}$ However, pregnant women with heart disease do remain at risk for other complications including heart failure, arrhythmia, and stroke. Women with congenital heart disease now comprise the majority of pregnant women with heart disease seen at referral centres. The next largest group includes women with rheumatic heart disease. Peripartum cardiomyopathy, though infrequent, will be discussed in view of its unique relation to pregnancy. Two groups of conditions not discussed further are coronary artery disease, infrequently encountered, and isolated mitral valve prolapse, which generally has an excellent outcome.

\section{Cardiovascular physiology and pregnancy}

Hormonally mediated increases in blood volume, red cell mass, and heart rate result in a major increase in cardiac output during pregnancy; cardiac output peaks during the second trimester, and remains constant until term. Gestational hormones, circulating prostaglandins, and the low resistance vascular bed in the placenta result in concomitant decreases in peripheral vascular resistance and blood pressure. During labour and delivery, pain and uterine contractions result in additional increases in cardiac output and blood pressure. Immediately following delivery, relief of caval compression and autotransfusion from the emptied and contracted uterus produce a further increase in cardiac output. Most haemodynamic changes of pregnancy resolve by two weeks postpartum.

\section{Outcomes associated with specific} cardiac lesions

Correspondence to: Samuel Siu MD, PMCC 3-526, Toronto General Hospital, 200 Elizabeth Street, Toronto, Ontario M5G 2C4, Canada Sam.Siu@uhn.on.ca septal defect (VSD) and patent ductus arteriosus, is counterbalanced by the decrease in peripheral vascular resistance. Consequently, the

\section{Congenital heart lesions}

Left to right shunts

The effect of increase in cardiac output on the increase in volume overload is attenuated. In the absence of pulmonary hypertension, pregnancy, labour and delivery are well tolerated. ${ }^{24}$ However arrhythmias, ventricular dysfunction, and progression of pulmonary hypertension may occur, especially when the shunt is large or when there is pre-existing elevation of pulmonary artery pressure. Infrequently, particularly in ASDs, paradoxical embolisation may be encountered if systemic vasodilatation and/or elevation of pulmonary resistance promote transient right to left shunting.

\section{Left ventricular outflow tract obstruction}

When aortic stenosis complicates pregnancy it is usually because of congenital bicuspid aortic valve which may also be associated with aortic coarctation and/or ascending aortopathy. Other causes of left ventricular outflow tract obstruction at, below, and above the valve have similar haemodynamic consequences. Women with symptomatic aortic stenosis should delay pregnancy until after surgical correction. ${ }^{6}$ However the absence of symptoms antepartum is not sufficient assurance that pregnancy will be well tolerated. In a pregnant woman with severe aortic stenosis, the limited ability to augment cardiac output may result in abnormal elevation of left ventricular systolic and filling pressures which in turn precipitate or exacerbate heart failure or ischaemia. In addition the non-compliant, hypertrophied ventricle is sensitive to falls in preload. The consequent exaggerated drop in cardiac output may lead to hypotension. In a compilation of many small retrospective series, 65 patients were followed through 106 pregnancies with a maternal mortality of $11 \%$ and a perinatal mortality of $4 \% .^{7}$ Most of the complications were reported in the earlier studies. In 25 pregnancies managed more recently, there was no maternal mortality but deterioration of maternal functional status occurred in $5(20 \%))^{7}$ Intrapartum palliation by balloon valvuloplasty may be helpful in selected cases.

In the absence of prosthetic dysfunction or residual aortic stenosis, patients with bioprosthetic aortic valves usually tolerate pregnancy well. Though it had been stated that pregnancy might accelerate the rate of degeneration of bioprosthetic or homograft valves, recent studies have shown that this is not the case. ${ }^{8}$ A study of 14 pregnancies in women who underwent pulmonary autograft aortic valve replacement (Ross procedure) reported favourable maternal and fetal outcomes except in one woman who developed postpartum left ventricular dysfunction. ${ }^{9}$ Pregnancy in a woman with a mechanical valve prosthesis carries increased risk of valve thrombosis as a result of the hypercoagulable state. The magnitude of this increased risk $(3-14 \%)$ is greater if subcutaneous unfractionated heparin rather than warfarin is used as the anticoagulant agent and may be a result of inadequate dosing, monitoring or reduced efficacy with subcutaneous unfractionated heparin. ${ }^{6}{ }^{10}$ 
Coarctation of the aorta

Maternal mortality with uncorrected coarctation has been reported as 3\% in an early series, and is higher in the presence of associated cardiac defects, aortopathy, or longstanding hypertension; aortic rupture accounted for eight of the 14 reported deaths and occurred in the third trimester as well as in the postpartum period. ${ }^{11}$ More recently, a preliminary report described encouraging maternal and fetal outcome in 87 pregnancies, with no maternal deaths and one early neonatal death. ${ }^{12}$ The management of hypertension in uncorrected coarctation is particularly problematic in pregnancy because satisfactory control of upper body hypertension may lead to excessive hypotension below the coarctation site, compromising the fetus. Intrauterine growth restriction and premature labour and delivery are more common. Following coarctation repair, the risk of dissection and rupture is likely reduced but not eliminated.

\section{Pulmonary stenosis}

Mild pulmonic stenosis, or pulmonic stenosis that has been alleviated by valvuloplasty or surgery, is well tolerated during pregnancy and fetal outcome is favourable. Though a woman with severe pulmonic stenosis may be asymptomatic, the increased haemodynamic load of pregnancy may precipitate right heart failure or atrial arrhythmias; such a patient should be considered for correction before pregnancy. Even during pregnancy, balloon valvuloplasty may be feasible if symptoms of pulmonary stenosis progress.

Cyanotic heart disease: unrepaired and repaired In uncorrected or palliated pregnant patients with cyanotic congenital heart disease such as tetralogy of Fallot, single ventricle, etc, the usual pregnancy associated fall in systemic vascular resistance and rise in cardiac output exacerbate right to left shunting leading to increased maternal hypoxaemia and cyanosis. A recent report examining the outcomes of 96 pregnancies in 44 women with a variety of cyanotic congenital heart defects reported a high rate of maternal cardiac events (32\%, including one death), prematurity (37\%), and a low live birth rate $(43 \%) .{ }^{13}$ The lowest live birth rate $(12 \%)$ was observed in those mothers with an arterial oxygen saturation of $\leqslant 85 \%$.

Tetralogy of Fallot is the most common form of cyanotic congenital heart disease. Pregnancy risk is low in women who have had successful correction of tetralogy. ${ }^{24}$ However, residua and sequelae such as residual shunt, right ventricular outflow tract obstruction, arrhythmias, pulmonary regurgitation, right ventricular systolic dysfunction, pulmonary hypertension (caused by the effects of a previous palliative shunt), or left ventricular dysfunction (caused by previous volume overload) increase the likelihood of pregnancy complications and require independent consideration.

Atrial repair (Mustard or Senning procedure) was developed for the surgical correction of complete transposition of the great arteries.
The anatomic right ventricle supports the systemic circulation. Late adult complications following atrial repair include sinus node dysfunction, atrial arrhythmias, and dysfunction of the systemic ventricle. In 43 pregnancies in 31 women described in recent reports, there was one late maternal death. ${ }^{14}$ There was a $14 \%$ incidence of maternal heart failure, arrhythmias, or cardiac deterioration. Few recipients of the current repair of choice for complete transposition - the arterial switch procedurehave yet reached reproductive age.

The Fontan operation eliminates cyanosis and volume overload of the functioning systemic ventricle but patients have a limited ability to increase cardiac output. In a recent review of 33 pregnancies in 21 women who were doing well after the Fontan operation, there were $15(45 \%)$ term pregnancies with no maternal mortality although two women had cardiac complications and the incidence of first trimester miscarriage was high $(39 \%) .{ }^{15}$ Since the 10 year survival rate following the Fontan operation is only $60-80 \%$, it is important that information regarding long term maternal prognosis be discussed during preconception counselling.

\section{Marfan syndrome}

Life threatening aortic complications of Marfan syndrome are caused by medial aortopathy resulting in dilatation, dissection, and valvar regurgitation. Risk is increased in pregnancy because of haemodynamic stress and perhaps hormonal effects. Although older case reports suggested a very high mortality risk in the range of $30 \%$, more recent data suggested an overall maternal mortality of $1 \%$ and fetal mortality of $22 \%$. A prospective study of 45 pregnancies in 21 patients reported no increase in obstetrical complications or significant change in aortic root size in the patients with normal aortic roots. Importantly, in the eight patients with a dilated aortic root $(>40 \mathrm{~mm})$ or prior aortic root surgery, three of their nine pregnancies were complicated by either aortic dissection (two) or rapid aortic dilatation (one). ${ }^{16}$ Thus, patients with aortic root involvement should receive preconception counselling emphasising their risk, and in early pregnancy should be offered termination. In contrast, women with little cardiovascular involvement and with normal aortic root diameter may tolerate pregnancy well, though there remains a possibility of dissection even without prior evidence of aortopathy. Serial echocardiography should be used to identify progressive aortic root dilatation and prophylactic $\beta$ blockers should be administered.

\section{Congenitally corrected transposition of the great} arteries

Many adult patients will have had surgical interventions, primarily VSD closure and relief of pulmonic stenosis, sometimes requiring a valved conduit from the left ventricle to the pulmonary artery. Potential problems in pregnancy include dysfunction of the systemic right ventricle and/or increased systemic atrioventricular valve regurgitation with heart failure, 
atrial arrhythmias, and atrioventricular block. In two recent reports on 41 patients, there were 105 pregnancies with $73 \%$ live births and no maternal mortality, although seven patients developed either heart failure, endocarditis, stroke, or myocardial infarction. ${ }^{17} 18$

\section{Eisenmenger syndrome and pulmonary vascular} obstructive disease

Maternal mortality in Eisenmenger syndrome is approximately $30 \%$ in each pregnancy. ${ }^{19}$ The preponderance of complications occurs at term and during the first postpartum week. Preconception counselling should stress the extreme pregnancy associated risks. Termination of pregnancy should always be offered to such patients, as should sterilisation. The vasodilation associated with pregnancy will increase the degree of right to left shunting in patients with Eisenmenger syndrome, resulting in worsening of maternal cyanosis with adverse effect on fetal outcome. Spontaneous abortion is common, intrauterine growth restriction is seen in $30 \%$ of pregnancies, and preterm labour is frequent. The high perinatal mortality rate $(28 \%)$ is caused mainly by prematurity.

A recent review of outcome of 125 pregnancies in patients with Eisenmenger syndrome, primary pulmonary hypertension, and secondary pulmonary hypertension reported poor outcomes in all three groups. ${ }^{20}$ The maternal mortality observed in the Eisenmenger, primary, and secondary pulmonary hypertension groups was $36 \%, 30 \%$, and $56 \%$, respectively. The overall neonatal mortality was $13 \%$.

\section{Rheumatic heart disease}

Mitral stenosis is the most common rheumatic valvar lesion encountered during pregnancy. The hypervolaemia and tachycardia associated with pregnancy exacerbate the impact of mitral valve obstruction. The resultant elevation in left atrial pressure increases the likelihood of atrial fibrillation. Thus, even patients with mild to moderate mitral stenosis, who are asymptomatic before pregnancy, may develop atrial fibrillation and heart failure during the anteand peripartum periods. Atrial fibrillation is a frequent precipitant of heart failure in pregnant patients with mitral stenosis, primarily caused by uncontrolled ventricular rate, and equivalent tachycardia of any cause may produce the same detrimental effect. Earlier studies examining a pregnant population comprised predominantly of women with rheumatic mitral disease showed that mortality rate increased with worsening antenatal maternal functional class. $^{3}$ A more recent study found no mortality but described substantial morbidity from heart failure and arrhythmia. ${ }^{4}$

Pregnant women whose dominant lesion is rheumatic aortic stenosis have a similar outcome to those with congenital aortic stenosis. Severe aortic or mitral regurgitation is generally well tolerated during pregnancy although deterioration in maternal functional class has been observed.

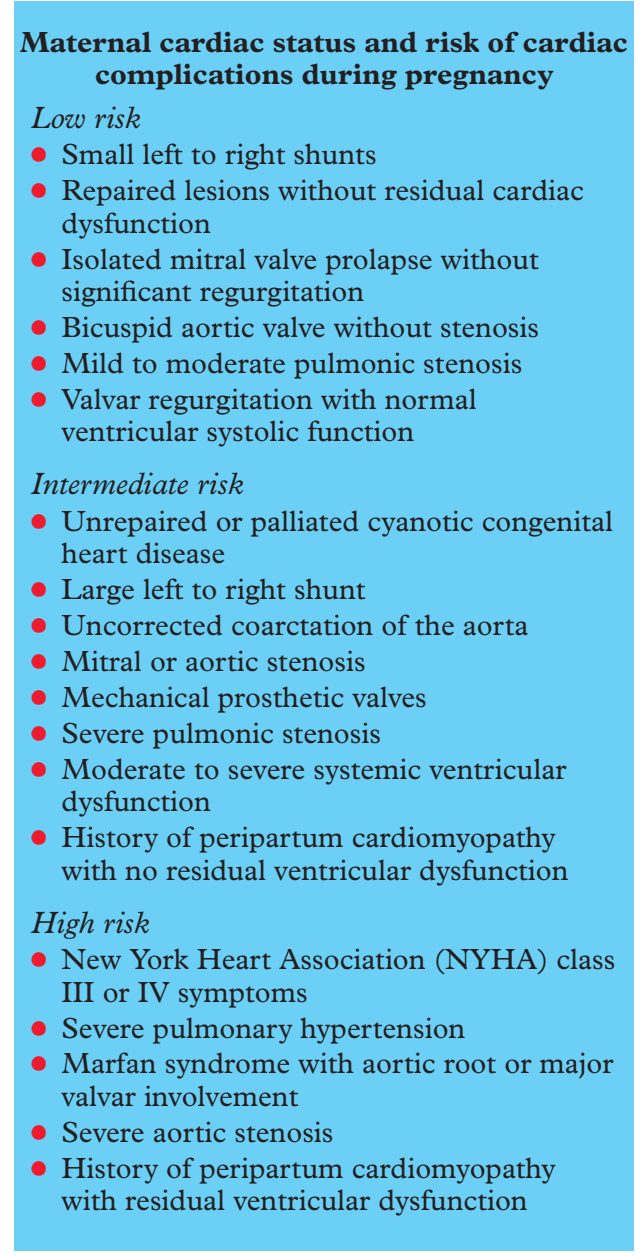

Peripartum cardiomyopathy

Peripartum cardiomyopathy is a form of idiopathic dilated cardiomyopathy diagnosed by otherwise unexplained left ventricular systolic dysfunction, confirmed echocardiographically, presenting during the last antepartum month or in the first five postpartum months. ${ }^{21}$ It usually manifests as heart failure, although arrhythmias and embolic events also occur. Many affected women will show improvement in functional status and ventricular function postpartum, but others may have persistent or progressive dysfunction. The relapse rate during subsequent pregnancies is substantial in women with evidence of persisting cardiac enlargement or left ventricular dysfunction. It remains unclear whether pregnancy is safe in those with recovery of systolic function. Dobutamine stress echocardiography may have a role in evaluating contractile reserve in women with recovered systolic function who are contemplating further pregnancies, but there are, as yet, insufficient data to confirm the validity of this approach.

\section{Management}

Risk stratification and counselling

Risk stratification and counselling of women with heart disease is best accomplished before 
conception. The data required for risk stratification can be acquired readily from a thorough cardiovascular history and examination, 12 lead ECG, and transthoracic echocardiogram. In patients with cyanosis, arterial oxygen saturation should be assessed by percutaneous oximetry. In counselling, the following six areas should be considered: the underlying cardiac lesion, maternal functional status, the possibility of further palliative or corrective surgery, additional associated risk factors, maternal life expectancy and ability to care for a child, and the risk of congenital heart disease in offspring.

Defining the underlying cardiac lesion is an important part of stratifying risk and determining management. The nature of residua and sequelae should be clarified, especially ventricular function, pulmonary pressure, severity of obstructive lesions, persistence of shunts, and presence of hypoxaemia. Almost all patients can be stratified into low, intermediate, or high risk groups. Maternal functional status is widely used as a predictor of outcome, and most often defined by New York Heart Association (NYHA) functional class. In a study of 482 pregnancies in women with congenital heart disease, cardiovascular morbidity was less $(8 \% v 30 \%)$ and live birth rate higher $(80 \% v 68 \%)$ in mothers with NYHA functional class I compared to the others. ${ }^{1} \mathrm{We}$ recently examined 276 pregnancies in 221 women and showed that poor functional status (NYHA > II) or cyanosis, myocardial dysfunction, left heart obstruction, prior arrhythmia, and prior cardiac events were independent predictors of maternal cardiac complications. ${ }^{4}$ Poor maternal functional class or cyanosis was also predictive of adverse neonatal events.

Further palliative or corrective surgery. Both maternal and fetal outcomes are improved by surgery to correct cyanosis, which should be undertaken before conception when possible. Similarly, patients with symptomatic obstructive lesions should undergo intervention before pregnancy. A systematic overview of cardiovascular surgical outcomes during pregnancy reported a maternal and fetal mortality of $6 \%$ and $30 \%$, respectively. ${ }^{22}$ Valve replacement requires weighing the need for ongoing anticoagulation with a mechanical valve against the likelihood of early reoperation if a tissue valve is used. For aortic stenosis, an attractive alternative is the pulmonary autograft.

Additional associated risk factors that may complicate pregnancy include a history of arrhythmia or heart failure, prosthetic valves and conduits, anticoagulant treatment, and the use of teratogenic drugs such as warfarin or angiotensin converting enzyme inhibitors.

Maternal life expectancy and ability to care for her child. A patient with limited physical capacity or with a condition that may result in premature maternal death should be advised of her potential inability to look after her child. Women whose condition imparts a high likelihood of fetal complications, such as those with cyanosis or on anticoagulants, must be apprised of these added risks.

The risk of recurrence of congenital heart disease in offspring should be addressed in the context of a $0.4-0.6 \%$ risk in the general population. The risk with a first degree relative affected increases about 10-fold. Left heart obstructive lesions have a higher recurrence rate. Certain conditions such as Marfan syndrome and the 22q11 deletion syndromes are autosomal dominant, conferring a $50 \%$ risk of recurrence in an offspring. Patients with congenital heart disease who reach reproductive age should be offered genetic counselling so that they are fully informed of the mode of inheritance and recurrence risk as well as the prenatal diagnosis options available to them. Preventive strategies to decrease the incidence of congenital defects such as preconception use of multivitamins containing folic acid can be discussed at the time of such counselling. ${ }^{23}$

\section{Antepartum management}

Pregnant women with heart disease may be at particular risk for one or more of congestive heart failure, arrhythmias, thrombosis, emboli, and adverse effects of anticoagulants.

When ventricular dysfunction is a concern, activity limitation is helpful and in severely affected women with NYHA class III or IV symptoms, hospital admission by mid second trimester may be advisable. Pregnancy induced hypertension, hyperthyroidism, infection, and anaemia should be identified early and treated vigorously. For patients with important mitral stenosis, the use of $\beta$ blockers or digoxin for control of heart rate should be considered. We also offer empiric treatment with $\beta$ blockers to patients with coarctation and to Marfan patients.

\section{Arrhythmias}

Arrhythmias in the form of premature atrial or ventricular beats are common in normal pregnancy, although sustained tachyarrhythmias have also been reported. In those with pre-existing arrhythmias, pregnancy may exacerbate their frequency or haemodynamic severity. Pharmacological treatment is usually reserved for patients with severe symptoms or when sustained episodes are poorly tolerated in the presence of ventricular hypertrophy, ventricular dysfunction, or valvar obstruction. Sustained tachyarrhythmias such as atrial flutter or atrial fibrillation should be treated promptly, avoiding teratogenic antiarrhythmic drugs. Digoxin and $\beta$ blockers are antiarrhythmic drugs of choice in view of their known safety profiles. ${ }^{24}$ Quinidine, adenosine, sotalol, and lidocaine are also "safe" but published data on their use during pregnancy is more limited. Amiodarone is more problematic and standard texts classify it as contraindicated in pregnancy; however, there are case reports describing successful use with careful follow up including assessment of neonatal thyroid function. Electrical cardioversion is safe in pregnancy. A recent report of 44 pregnancies in women with implantable cardioverterdefibrillators reported favourable maternal and fetal outcomes. ${ }^{25}$ 


\section{Anticoagulation}

When a pregnant woman with mechanical heart valve requires anticoagulation, heparin and warfarin are used but controversy continues as to which is better at different stages of pregnancy. Oral anticoagulation with warfarin is better accepted by patients, and is effective. However, warfarin embryopathy may be produced during organogenesis, and fetal intracranial bleeding can occur throughout pregnancy. A recent study of 58 pregnancies reported that a daily warfarin dose of $\leqslant 5 \mathrm{mg}$ was associated with no cases of embryopathy. ${ }^{10}$ Fetal intracranial haemorrhage during vaginal delivery is a risk with warfarin unless it has been stopped at least two weeks before labour. Adjusted dose subcutaneous heparin has no teratogenic effects, as the drug does not cross the placenta, but may cause maternal thrombocytopenia and osteoporosis. Claims of inadequate effectiveness of heparin in patients with mechanical heart valves have been countered by arguments that inadequate doses were used. Recent practice guidelines have favoured use of warfarin and low dose aspirin either during the entire pregnancy or substituted by heparin only during the peak teratogenic period (sixth to 12 th week of gestation). ${ }^{6}$ Low molecular weight heparin is easier to administer and has been suggested as an alternative to adjusted dose unfractionated heparin. ${ }^{26}$ Clinical trials examining the optimal anticoagulation strategy are needed.

\section{Eisenmenger syndrome}

If a woman with Eisenmenger syndrome does not accept counselling to terminate, or presents late in pregnancy, meticulous antepartum management is necessary including early hospitalisation, supplemental oxygen, and possibly empiric anticoagulation. There have been several case reports describing reduction of pulmonary pressure with the use of nitric oxide in pregnant patients. However, despite haemodynamic improvement, the maternal mortality in this small experience remained high.

\section{Multidisciplinary approach and high risk pregnancy units}

Women with heart disease who are at intermediate or high risk for complications should be managed in a high risk pregnancy unit by a multidisciplinary team from obstetrics, cardiology, anaesthesia, and paediatrics. When dealing with a complex problem the team should meet early in the pregnancy. At this time the nature of the cardiac lesion, anticipated effects of pregnancy, and potential problems are explored. Since it is often not possible for every member of the team to be at the patient's bedside at a moment of crisis, it is helpful to develop and distribute widely a written management plan for foreseeable contingencies. Women with heart disease in the "low risk" group can be managed in a community hospital setting. However, if there is doubt about the mother's status or the risk, consultation at a regional referral centre should be arranged.
Management of pregnancy complicated by maternal heart disease

\section{All patients}

- Define the lesion, the residua, and the sequelae

- Assess functional status

- Determine predictors of risk

- Eliminate teratogens

- Arrange genetic counselling when relevant

- Consider consultation with a regional centre

- Assess need for endocarditis prophylaxis during labour and delivery

Intermediate and high risk patients

- Arrange management at a regional centre for high risk pregnancy

- Consider antepartum interventions to reduce pregnancy risk

- Engage a multidisciplinary team, as appropriate

- Consider a multidisciplinary case conference

- Develop and disseminate a management plan

- Anticipate vaginal delivery in almost all cases, unless there are obstetrical contraindications

- Consider early epidural anesthesia

- Modify labour and delivery to reduce cardiac work

- Plan postpartum monitoring, sometimes in a coronary or intensive care unit setting

\section{Labour and delivery}

Vaginal delivery is recommended with very few exceptions. The only cardiac indications for caesarean section are aortic dissection, Marfan syndrome with dilated aortic root, and failure to switch from warfarin to heparin at least two weeks before labour. Preterm induction is rarely indicated, but once fetal lung maturity is assured a planned induction and delivery in high risk situations will ensure availability of appropriate staff and equipment. Although there is no consensus on the use of invasive haemodynamic monitoring during labour and delivery, we commonly utilise intra-arterial monitoring (with or without concurrent pulmonary artery catheterisation) in cases where there are concerns about the interpretation and deleterious effects of a sudden drop in systemic blood pressure (such as in patients with severe aortic stenosis, pulmonary hypertension, or more than moderate systemic ventricular systolic dysfunction). Placement of an indwelling pulmonary artery catheter should be considered only when the information sought is not available otherwise and warrants the risk of the procedure, which may be significant especially in the setting of pulmonary hypertension.

Heparin anticoagulation is discontinued at least 12 hours before induction, or reversed with protamine if spontaneous labour develops, and can usually be resumed 6-12 hours postpartum. 
Endocarditis prophylaxis is initiated at onset of active labour when indicated. The American Heart Association recommendations state that delivery by caesarean section and vaginal delivery in the absence of infection do not require endocarditis prophylaxis except, perhaps, in patients at high risk. However, many centres with extensive experience in caring for pregnant women with heart disease utilise endocarditis prophylaxis routinely, as an uncomplicated delivery cannot always be anticipated.

Epidural anaesthesia with adequate volume preloading is the technique of choice. Epidural fentanyl is particularly advantageous in cyanotic patients with shunt lesions as it does not lower peripheral vascular resistance. In the presence of a shunt, air and particulate filters should be placed in all intravenous lines.

Labour is conducted in the left lateral decubitus position to attenuate haemodynamic fluctuations associated with contractions in the supine position. Forceps or vacuum extraction will shorten the latter part of the second stage of labour and reduce need for maternal expulsive efforts. As haemodynamics do not return to baseline for many days after delivery, those patients at intermediate or high risk may require monitoring for a minimum of 72 hours postpartum. Patients with Eisenmenger syndrome require longer close postpartum observation, since mortality risk persists for up to seven days.

1. Whittemore R, Hobbins J, Engle M. Pregnancy and its outcome in women with and without surgical treatment of congenital heart disease. Am J Cardiol 1982;50:641-51. - The first large study examining pregnancy outcomes in women with congenital heart disease. The relation between maternal functional class and cardiac risk during pregnancy was demonstrated in this study.

2. Shime J, Mocarski E, Hastings D, et al. Congenital heart disease in pregnancy: short- and long-term

implications. Am J Obstet Gynecol 1987;156:313-22.

- This study, together with reference 1, provided much of the early risk stratification data on pregnancy outcomes in women with congenital heart disease.

3. McFaul P, Dornan J, Lamki H, et al. Pregnancy complicated by maternal heart disease. A review of 519 women. Br J Obstet Gynaecol 1988;95:861-7.

- Examining a patient population with predominantly rheumatic heart disease, this study observed a relation between maternal functional class and mortality risk during pregnancy.

4. Siu SC, Sermer M, Harrison DA, et al. Risk and predictors for pregnancy-related complications in women with heart disease. Circulation 1997;96:2789-94.

- This retrospective study assessed maternal and neonatal outcomes in a contemporary cohort of pregnant women with heart disease and developed a risk index which can be used to estimate the risk of cardiac complications during pregnancy.

website

extra

Additional references appear on the

Heart website

www.heartjnl.com
5. Hunter S, Robson SC. Adaptation of the maternal heart in pregnancy. Br Heart J 1992;68:540-3.

6. Bonow RO, Carabello B, de Leon AC, Jr, et al. Guidelines for the management of patients with valvular heart disease: executive summary. A report of the American College of Cardiology/American Heart Association task force on practice guidelines (committee on management of on practice guidelines (committee on management 1998;98:1949-84.
Recently published recommendations on the risk stratification and treatment of pregnant women with valvar heart lesions.

7. Lao T, Sermer M, MaGee L, et al. Congenital aortic stenosis and pregnancy-a reappraisal. Am J Obstet Gynecol 1993;169:540-5.

8. North RA, Sadler L, Stewart AW, et al. Long-term survival and valve-related complications in young women with cardiac valve replacements. Circulation

1999;99:2669-76.

9. Dore A, Somerville J. Pregnancy in patients with pulmonary autograft valve replacement. Eur Heart $J$ pulmonary autograt

10. Vitale N, De Feo M, De Santo LS, et al. Dose-dependent fetal complications of warfarin in pregnant women with mechanical heart valves. J Am Coll Cardiol 1999:33:1637-41.

11. Deal K, Wooley CF. Coarctation of the aorta and pregnancy. Ann Intern Med 1973;78:706-10.

12. Connolly H, Ammash N, Warnes C. Pregnancy in women with coarctation of the aorta [abstract]. J Am Coll Cardiol 1996;27:43A.

13. Presbitero P, Somerville J, Stone S, et al. Pregnancy in cyanotic congenital heart disease. Outcome of mother and fetus. Circulation 1994:89:2673-6.

- The largest case series to date examining the pregnancy outcomes of cyanotic women without pulmonary hypertension.

14. Genoni M, Jenni R, Hoerstrup SP, et al. Pregnancy after atrial repair for transposition of the great arteries. Heart 1999:81:276-7.

15. Canobbio M, Mair D, van der Velde $\mathbf{M}$, et al Pregnancy outcomes after the Fontan repair. J Am Coll Cardiol 1996;28:763-7.

16. Rossiter J, Repke J, Morales A, et al. A prospective longitudinal evaluation of pregnancy in the Marfan syndrome. Am J Obstet Gynecol 1995;173:1599-606.

17. Connolly HM, Grogan M, Warnes CA. Pregnancy among women with congenitally corrected transposition of great arteries. J Am Coll Cardiol 1999;33:1692-5.

18. Therrien J, Barnes I, Somerville J. Outcome of pregnancy in patients with congenitally corrected transposition of the great arteries. Am J Cardiol 1999;84:820-4

19. Gleicher N, Midwall J, Hochberger D, et al. Eisenmenger's syndrome and pregnancy. Obstet Gynecol Surv 1979;34:721-41.

20. Weiss B, Zemp L, Seifert B, et al. Outcome of pulmonary vascular disease in pregnancy: a systematic overview from 1978 through 1996. J Am Coll Cardiol 1998;31:1650-7.

- A comprehensive overview of pregnancy outcomes in women with pulmonary hypertension.

21. Pearson GD, Veille JC, Rahimtoola S, et al Peripartum cardiomyopathy: National Heart, Lung, and Blood Institute and Office of Rare Diseases (National Institutes of Health) workshop recommendations and review. JAMA 2000;283:1183-8.

- A comprehensive overview on the current state of the art knowledge in this area.

22. Weiss BM, von Segesser LK, Alon E, et al. Outcome of cardiovascular surgery and pregnancy: a systematic review of the period 1984-1996. Am J Obstet Gynecol 1998;179:1643-53.

23. Czeizel A. Reduction of urinary tract and cardiovascular defects by periconceptional multivitamin supplementation. Am J Med Genet 1996;62:179-83.

24. Chow T, Galvin J, McGovern B. Antiarrhythmic drug therapy in pregnancy and lactation. Am J Cardiol

1998;82:581-62

- A comprehensive overview of the use of antiarrhythmic agents during pregnancy.

25. Natale A, Davidson T, Geiger M, et al. Implantable cardioverter-defibrillators and pregnancy: a safe

combination? Circulation 1997;96:2808-12.

26. Ginsberg JS, Hirsh J. Use of antithrombotic agents during pregnancy. Chest 1998:114:524S-30S.

Practice guidelines that incorporate the conflicting opinions and data regarding the choice of anticoagulation regimen during pregnancy. 